\title{
Effects of menopause on intraindividual changes in serum lipids, blood pressure, and body weight - the Chin-Shan community cardiovascular cohort study
}

\author{
Pao-Ling Torng a , Ta-Chen $\mathrm{Su}^{\mathrm{b}}$, Fung C. Sung ${ }^{\mathrm{c}}$, Kuo-Liong Chien ${ }^{\mathrm{b}}$, \\ Su-Cheng Huang a, Song-Nan Chow a, Yuan-Teh Lee ${ }^{b, *}$ \\ ${ }^{a}$ Department of Obstetric and Gynecology, National Taiwan University College of Medicine, Taipei 10020, Taiwan, ROC \\ ${ }^{\mathrm{b}}$ Department of Internal Medicine, National Taiwan University College of Medicine, No. 7, Chungshan South Road, Taipei, Taiwan, ROC \\ ${ }^{\mathrm{c}}$ Institute of Environmental Health, National Taiwan University College of Public Health, Taipei, Taiwan, ROC
}

Received 13 March 2001; received in revised form 24 June 2001; accepted 10 July 2001

\begin{abstract}
In Taiwan, the Chin-Shan Community Cardiovascular Cohort (CCCC) was assessed prospectively to determine whether the changes in cardiovascular risk factors for women age 45-54 years are due to menopause. The average paired percentage changes that occurred between baseline (1990-1991) and follow-up (4 years later) in fasting serum lipids were compared in three groups of women including groups of 59 and 224 who were pre- and postmenopausal, respectively, and a group of 118 who had spontaneously stopped menstruating. Postmenopausal women had the least gain in body mass index (BMI), whereas, mainly premenopausal women had increased systolic blood pressure $(P<0.05)$. All women had elevated total cholesterol (TC) levels, with the greatest elevation in women transitioning into menopause $(P<0.001)$. Low-density lipoprotein cholesterol (LDL-C) levels increased before and during the transition to menopause, but decreased after menopause $(P<0.01)$. Age had significant association with changes in TC, triglyceride (TG) and LDL-C levels, whereas BMI had significant association with changes in TG, LDL-C, and high-density lipoprotein cholesterol (HDL-C) levels $(P<0.05)$. After controlling for age and BMI, only differences in TC remained significant, with the greatest gain in women who stopped menstruating $(12.9 \%)$ followed by pre- $(6.5 \%)$ and postmenopausal women (4.8\%). Changes in both systolic and diastolic blood pressures, and TG and HDL-C levels were not significantly different, but HDL-C levels declined between 11.5 and $14.7 \%$ in all groups. This study suggests an unfavorable effect of menopause on lipid metabolism, especially on the TC level, which was predominantly elevated during the transition to menopause. The decline of HDL-C is of concern. (C) 2002 Elsevier Science Ireland Ltd. All rights reserved.
\end{abstract}

Keywords: Menopause; Transition; Lipids; Lipoproteins; Taiwanese women

\section{Introduction}

Numerous studies consider menopause a risk factor for coronary heart disease (CHD) [1,2] and suggest that postmenopausal women have atherogenic risks. Several cross-sectional studies found higher plasma levels of total cholesterol (TC), low-density lipoprotein choles-

\footnotetext{
* Corresponding author. Tel.: +886-2-23562000; fax: + 886-223217522 .

E-mail address: ytlee@ha.mc.ntu.edu.tw (Y.-T. Lee).
}

terol (LDL-C), and triglycerides (TG) in postmenopausal women [3-7]. The association between menopause and changes in these cardiovascular risk factors is difficult to study because menopausal status and age are correlated. Aging has been associated with increased TC, TG and LDL-C levels, and the rises of these lipids were particularly marked at the onset of menopause, including Asian women [8,9]. In a recent age-matched cross-sectional study, menopausal women (compared with premenopausal women) appear to have significantly higher TC, LDL-C but not HDL-C levels 
[10]. Our previous cross-sectional study based on the baseline data collected from the Chin-Shan Community Cardiovascular Cohort (CCCC) in 1990-1991 also showed atherogenic lipids were higher in postthan in premenopausal women. The postmenopausal increases were particularly significant in TC and LDL-C among women age 45-49 years, and in TG and Apo B among women age 50-54 years [11].

Several longitudinal studies focusing on the lipid changes in relation to menopause also have been carried out. An age-matched 2.5-year follow-up study found that women with natural menopause have increased LDL-C and decreased HDL-C levels, but unchanged blood pressure and body weight [12]. An earlier prospective study of Swedish women who stop menstruating showed intraindividual increases of TC, TG and body weight but not blood pressure [13]. They found, on the other hand, a significant increase of blood pressure in women with no change of menopausal status during the 6-year follow-up. The Nagasaki study showed a significantly increasing trend for serum cholesterol levels in both natural and surgically menopausal Japanese women, whereas no trend existed for systolic blood pressure (SBP) or body weight [14]. The study of Poehlman et al. [15], however, showed no effect on TC in menopausal women during a 6-year follow-up, although they did observe elevated LDL-C and TG but lowered HDLC. Ko et al. [9] suggested that the onset of menopause might further increase CVD risk for Chinese women. The CHD mortality rate has been fairly static in Taiwan [16], justifying the conduct of longitudinal studies to clarify the correlation of menopause to the age-related cardiovascular risk factors in women.

Most of the above-mentioned longitudinal studies compared CVD risk factor changes for individuals between pre- and postmenopause. Changes associated with the confounding effect of age were few and were measured by comparing women whose menopausal status changed with those whose status was unchanged during a similar follow-up period. In our previous cross-sectional study based on CCCC data, the age-associated changes in lipid levels were assumed to be constant regardless of menopausal status. The CCCC has been followed-up since 1990 to compare the absolute average intraindividual percentage changes between the baseline and succeeding measurements of body weight, blood pressure, lipid and lipoprotein levels in women who either remained premenopausal, spontaneously stopped menstruation, or became postmenopausal during the follow-up period. Less confounded by the effect of aging, this method of observation is more sensitive and able to demonstrate changes of lipids at menopause.

\section{Patients and methods}

\subsection{Study population}

The CCCC study was established in $1990-1991$ as previously reported [11,18]. In brief, the study cohort consisted of 3602 adults, age 35 and above, residing in a suburban community 20 miles north of metro Taipei. The mortality pattern in Chin-Shan was generally similar to that of the general Taiwanese population [16]. Among 1899 female respondents, 428 age 45-54 years were included in the baseline survey in 1990-1991, and 401 returned and completed the follow-up study 4 years later in 1994-1995. Women excluded from this report were those with hormone replacement therapy prior to or during the follow-up study period. Women with a history of diabetes, fasting sugar $\geq 140 \mathrm{mg}$, hysterectomy, oophorectomy, oral contraceptive pill use, or menopause before the age of 45 years also were not included in this study. Only subjects with secondary amenorrhea for at least 1 year were defined as menopausal. Subjects whose last menstruation was 311 months earlier were excluded because of uncertainty in their menopausal status.

\subsection{Data collection and assays}

The study team consisted of several cardiologists, senior residents, senior medical students, two assistant nurses and local practitioners. Trained medical students canvassed door-to-door with the assistance of community leaders to extend invitations for the baseline and biannual follow-up surveys. Written consent was obtained during the face-to-face questionnaire interview, and physicians later conducted physical examinations and laboratory tests on participants invited to the clinic established in the community. Specimens for blood analyses were also collected at the clinic.

All venous blood samples were taken after a $10-\mathrm{h}$ overnight fast, immediately refrigerated at $4{ }^{\circ} \mathrm{C}$, and transported to the National Taiwan University Hospital within $6 \mathrm{~h}$. Assay of serum TC and TG levels were normally performed within $24 \mathrm{~h}$. Otherwise, the serum samples were stored at $-70{ }^{\circ} \mathrm{C}$ for batch assay of LDL-C and HDL-C as previously described $[11,17,18]$. In brief, standard enzymatic methods for serum TC and TG were used (Merck 14354 and 14366, Germany, respectively). The HDL-C concentration in the supernatant was measured after precipitation with magnesium chloride phosphotungstate reagents (Merck 14993). The concentration of LDL-C was calculated as 'total cholesterol minus cholesterol in the supernatant,' and LDL-C precipitated by heparin-citrate reagent (Merck 14992).

Body mass index (BMI), an indicator related to the amount of visceral fat, was used as a measure of obesity 
and was calculated as body weight in $\mathrm{kg}$ divided by height in $\mathrm{m}^{2}$; these parameters were measured at the time of blood sampling. Blood pressure measurements were performed with mercury sphygmomanometer in a standardized fashion, cuff size adjusted to the circumference of the arm, and the arm placed with the cuff at the level of heart. Blood pressure was recorded using the mean of two measurements taken after 5 min of rest in the supine position. Systolic and diastolic blood pressures were defined according to Korotkoff sounds I and $\mathrm{V}$.

\subsection{Statistical analysis}

To distinguish the effect of menopause, data analyses emphasized comparing paired differences in serum lipids, body weight and blood pressure between the baseline and follow-up levels, among the three groups of study women identified at the time of follow-up survey. In doing so, average relative percentage changes in each factor between the two surveys were calculated for each individual, expressed in mean \pm S.E.M. These changes were also analyzed using paired $t$-test. A multivariate regression analysis was further conducted controlling for age and BMI as dependent variables. All statistical analyses were performed with SAS statistical software (Version 6.12, SAS Institute, Cary NC, USA). $P$ values below 0.05 were considered statistically significant.

\section{Results}

Of the 401 women from whom both baseline and follow-up data was collected, 59 women were still premenopausal, 118 had stopped menstruating (designated as 'transition' in the Figure and Tables) and 224 were postmenopausal during the 4-year follow-up period. The baseline average ages (S.D.) were 47.2 (1.8) years for women who remained premenopausal, 48.8 (2.5) years for those who became menopausal and $51.5(2.6)$ years for those who were postmenopausal (Fig. 1).
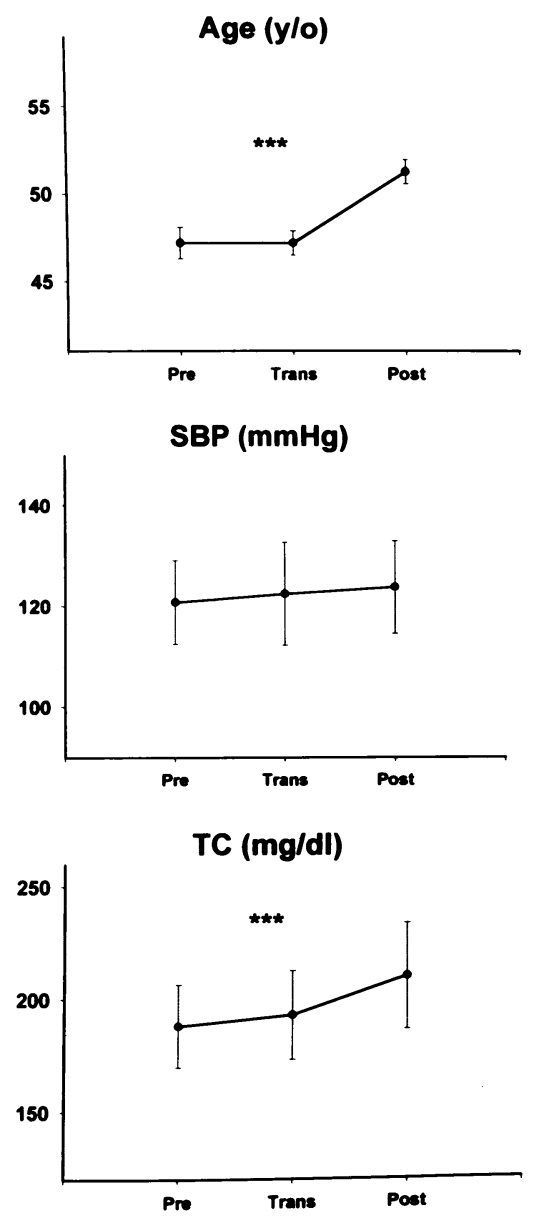
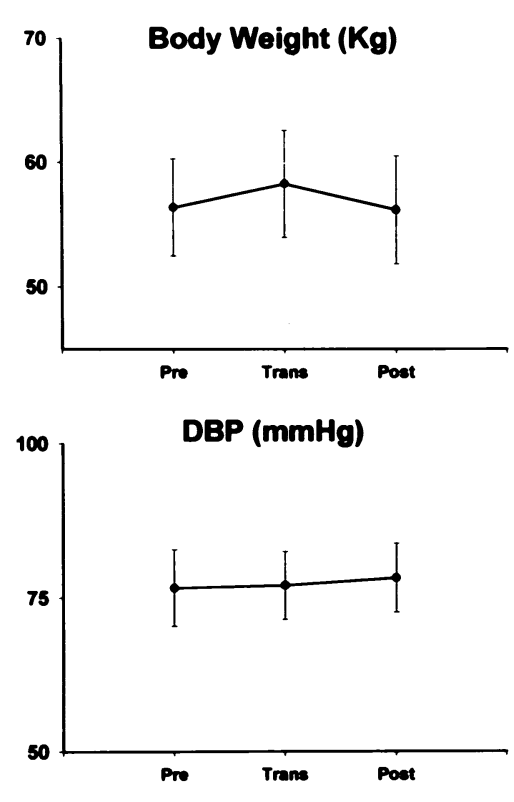

LDL-C (mg/dl)

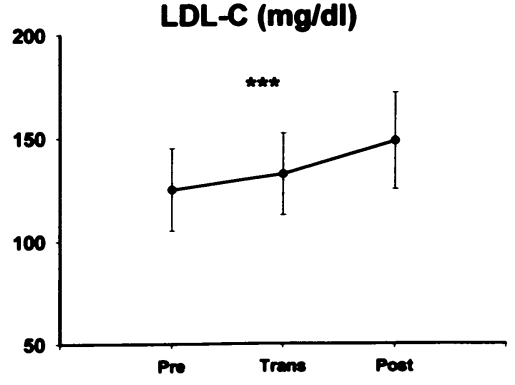

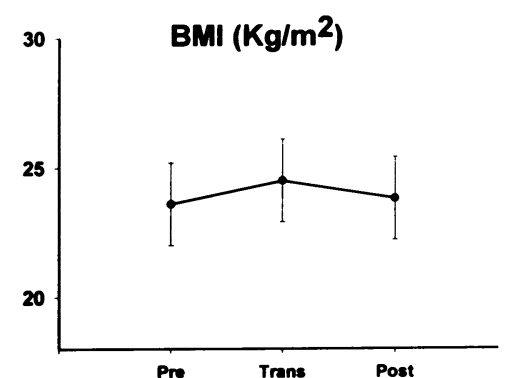
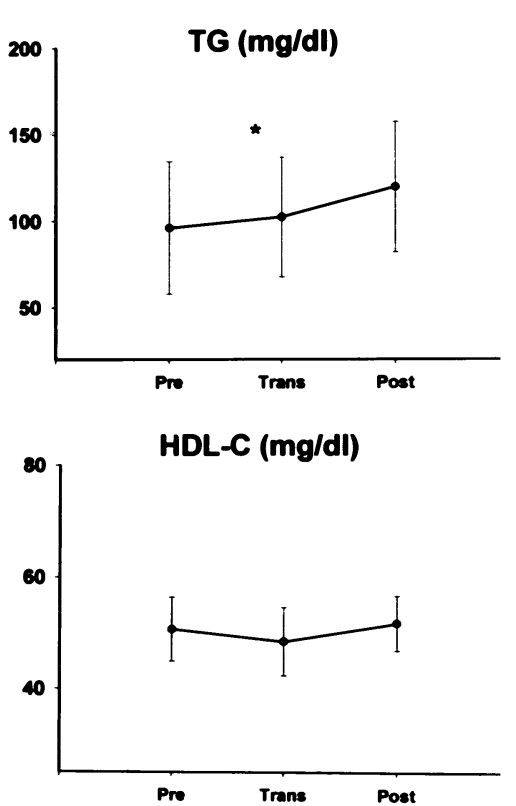

Fig. 1. Average baseline measurements of body weight, body mass index, blood pressure and lipids in Chin-Shan women categorized by menopausal status at the time of follow-up survey. Abbreviations: BP, blood pressure (mmHg); TC, total cholesterol (mg/dl); TG, triglyceride $(\mathrm{mg} / \mathrm{dl})$; LDL-C, low-density lipoprotein cholesterol (mg/dl); Pre, premenopause; Trans, transition from premenopause to postmenopause; post, postmenopause at follow-up. Data are expressed as the mean \pm S.D. $P=P$-value for difference among pre-, peri- and postmenopausal groups ( $t$-test). ${ }^{*} P<0.05,{ }^{* *} P<0.01,{ }^{* * *} P<0.001$ at the baseline. 
Table 1

Average paired percentage changes occurring between baseline and follow-up surveys of pre-, peri- and postmenopausal women in Chin-Shan Community Cardiovascular Cohort (CCCC)

\begin{tabular}{|c|c|c|c|c|}
\hline & Premenopause $(n=59)$ & Transition $(n=118)$ & Postmenopause $(n=224)$ & $P$ \\
\hline BMI & $4.8 \pm 1.4$ & $4.3 \pm 1.0$ & $1.5 \pm 0.8$ & $<0.05$ \\
\hline Body weight & $1.7 \pm 1.3$ & $2.3 \pm 0.9$ & $1.1 \pm 0.7$ & 0.59 \\
\hline SBP & $6.3 \pm 1.8$ & $2.1 \pm 1.3$ & $0.4 \pm 0.9$ & $<0.05$ \\
\hline DBP & $1.2 \pm 2.2$ & $3.5 \pm 1.5$ & $-0.4 \pm 1.1$ & 0.11 \\
\hline $\mathrm{TC}$ & $9.6 \pm 3.0$ & $14.2 \pm 2.2$ & $3.3 \pm 1.6$ & $<0.001$ \\
\hline TG & $-3.8 \pm 7.9$ & $2.0 \pm 5.8$ & $-1.5 \pm 4.2$ & 0.81 \\
\hline LDL-C & $6.4 \pm 4.1$ & $3.5 \pm 3.1$ & $-5.9 \pm 2.2$ & $<0.01$ \\
\hline HDL-C & $-13.5 \pm 2.5$ & $-10.4 \pm 1.9$ & $-15.6 \pm 1.3$ & 0.08 \\
\hline
\end{tabular}

Data are expressed as the mean \pm S.E. of mean (SEM). $P=P$-value for differences among pre-, peri- and postmenopausal groups (general linear regression).

Fig. 1 also depicts the average baseline body weights, BMIs, blood pressures and lipid profiles among these three groups. Although women approaching menopause grew heavier, no significant differences in average body weight and BMI were found among the three groups. Levels of TC, TG and LDL-C showed a progressive elevation among the three groups $(P<0.05$ to 0.001 ) with the highest values all in postmenopausal women. Differences in blood pressure and HDL-C were minor among three groups.

Based on the average paired percentage changes between the baseline and the follow-up data, all women showed elevations in body weight, BMI, SBP and TC levels in 4 years (Table 1). All these changes were the smallest in postmenopausal women. Changes in BMI, SBP and LDL-C were the largest in premenopausal women $(P<0.05)$, and the change in TC was the largest in women approaching menopause $(P<0.001)$. Decline in HDL-C was observed among all groups.

When age and BMI were adjusted in multivariate analysis, results showed percentage changes of body weight, BMI, DBP, SBP and HDL-C were independent of age, however, changes of TC, SBP and DBP were independent of BMI (Table 2). Changes of TG and LDL-C were age- and BMI-dependent, and the change of TC was age-dependent $(P<0.05)$. However, only the changes of TC were significantly different among the three groups $(P<0.05)$, with women who were approaching menopause having the greatest elevation of TC (12.9\%). LDL-C levels remained elevated for preand perimenopausal women, and lowered in postmenopausal women. TG levels were increased in postmenopausal women but decreased in premenopausal women and unchanged in women approaching menopause in the follow-up period.

Table 2 also shows changes in HDL-C was independent of age, and all groups had declines in HDL-C levels. In both pre and postmenopausal women, HDL$\mathrm{C}$ declined $14.7,3.2 \%$ greater than the decline in women approaching menopause.

\section{Discussion}

\subsection{Changes of serum lipids with menopause}

Our previous analyses [11] based on cross-sectional baseline data in 1990-1991 showed that postmenopausal women had higher serum levels of TC, TG, LDL-C and Apo B than premenopausal women. Among the women 45-54 years old, younger postmenopausal women showed significantly higher TC and LDL-C levels than premenopausal women, whereas older postmenopausal women showed significantly higher levels of TG. These trends are likely age and BMI-associated. The changes in coronary heart disease risk factors affecting the CCCC women (followed up for 4 years) were compared based on their menopausal status at the end of follow-up, and this study focused on those changes attributed to menopause, obesity and aging.

Postmenopausal women appeared to have higher TC, TG and LDL-C levels than premenopausal women and those women who became menopausal during the follow-up period. All women had elevated TC levels and the average elevation was the greatest in women approaching menopause. This pattern persisted after controlling for age and BMI. Though Poehlman et al. reported in a prospective study that natural menopause had no effect on TC levels [15], our data parallels that of other studies, showing that TC levels increase 2-3 years before [14,19] and may continue to increase 6 years after menopause [19]. The increase in TC may be slower for postmenopausal women.

In our study, after age and BMI adjustment, the decreased TG levels in crude data reverted to increased levels in postmenopausal women in the follow-up period, and women approaching menopause showed no net changes in TG. An earlier study also found that TG level was unaffected by menopause [12].

The trend of LDL-C was opposite to the trend of TG in this study. Ushiroyama, et al. [8] found elevations of 
TG and LDL-C levels at perimenopausal levels, particularly just before the onset of menopause. Do et al. [20] found that, during the period of transition to menopause, LDL-C and TG levels increased and HDL$\mathrm{C}$ levels decreased. The consistent rising trend of LDL$\mathrm{C}$ as menopause approaches is similar to our study, but contradicts our finding that LDL-C declines postmenopausally. This trend is age- and BMI-associated and statistically insignificant after controlling for these two factors. Therefore, despite the decline of LDL-C in postmenopausal women, their LDL-C level remained the highest because their baseline level was higher.

A decline of estrogen levels during menopause has been suggested to be associated with the rise of TG and LDL-C levels [8]. However, recent studies did not show the relation between endogenous sex hormone and lipid levels in pre [21], peri [22], and postmenopausal women [23]. And the opposite patterns in TG and LDL-C levels in our study cannot be further explained solely by the possible decrease in estrogen levels as menopause approaches. The contrasting decline of LDL-C levels in postmenopausal groups and decline of TG levels in premenopausal groups suggests that changes in lipids and lipoproteins are probably multifactorial in origin. The continued decline in HDL-C level, regardless of menopausal status, is the most striking change. With a $14.7 \%$ decrease in HDL-C, the $12.9 \%$ increase in TC indicated higher risk for women at menopause. Significant association of HDL-C levels with cardiac function in Chinese patients has been reported in our population [24], suggesting that HDL-C is an important independent risk factor for CHD in Chinese women as they age regardless of menopausal status.

Most women in Chin-Shan community have active daily routines and they seldom use hormone replacement therapy. The few that were using hormone replacement were excluded from our study. Herbal medicines for menopausal complaints were seldom taken. Consumption of a diet that included lots of soy beans and their products, which contain phyto-estro- gens that possibly improve menopausal syndrome and prevent atherosclerosis [25], was uniform among women of every age, and so had little effect on the lipid profile changes that occurred as a result of menopausal status in this study. However, physician's visits during surveys might influence participants' life styles and modify their CHD risk factors [26].

\subsection{Blood pressure in premenopausal and postmenopausal women}

Chow et al. [27] found both systolic and diastolic blood pressures are higher in women after menopause than in men of the same age, suggesting that estrogen deficiency might influence the age-related increase in blood pressure. However, in this study, systolic blood pressure markedly increased in the women who remained premenopausal at follow-up. After age and BMI were adjusted, this trend remained but was no longer significant. Postmenopausal women or women who became menopausal had only minor SBP increase. Lindquist et al. [13] also found a similar pattern of SBP change in their 6-year follow-up study. Several reports did not find that the change of SBP with menopause was significant $[10,12,14]$, but the European study found SBP significantly increased after menopause [15]. Administration of estrogen after menopause either attenuated the increase of both systolic and diastolic blood pressures [28-30] or had no detectable effect [31,32]. After menopause, with the cessation of ovulation, several hormones were diminished other than estrogen [33]. Therefore, the effect of hormone profiles on blood pressure before and after menopause was probably different.

Menopause was reported to have no effect on DBP $[10,14,15]$. But Shelley et al. reported in a longitudinal study that DBP increases as BMI increases during menopausal transition [22]. We did not observe any effect of natural menopause, age and BMI on DBP. Diastolic blood pressure may thus be a unique value to evaluate individual risk of CHD.

Table 2

A baseline and follow-up surveys of pre-, peri- and postmenopausal women in Chin-Shan Community Cardiovascular Cohort (CCCC)

\begin{tabular}{|c|c|c|c|c|c|c|}
\hline & Premenopause $(n=59)$ & Transition $(n=118)$ & Postmenopause $(n=224)$ & $P$ & B1 (Age) & $B 2(B M I)$ \\
\hline BMI & $3.7 \pm 1.6$ & $4.3 \pm 1.1$ & $1.8 \pm 0.8$ & 0.20 & 0.01 & $0.08^{\mathrm{a}}$ \\
\hline Body weight & $1.5 \pm 1.4$ & $1.8 \pm 1.0$ & $0.8 \pm 0.7$ & 0.67 & 0.005 & $0.032^{\mathrm{a}}$ \\
\hline SBP & $4.6 \pm 2.0$ & $1.6 \pm 1.3$ & $1.1 \pm 1.0$ & 0.33 & 0.06 & 0.02 \\
\hline DBP & $-0.3 \pm 2.4$ & $3.0 \pm 1.6$ & $0.2 \pm 1.2$ & 0.29 & 0.05 & 0.15 \\
\hline $\mathrm{TC}$ & $6.5 \pm 3.2$ & $12.9 \pm 2.3$ & $4.8 \pm 1.7$ & $<0.05^{\mathrm{a}}$ & $0.21^{\mathrm{a}}$ & 0.04 \\
\hline TG & $-13.3 \pm 8.5$ & 0 & $2.5 \pm 4.5$ & 0.28 & $1.75^{\mathrm{a}}$ & $1.19^{\mathrm{a}}$ \\
\hline LDL-C & $1.5 \pm 4.4$ & $2.0 \pm 3.2$ & $-3.8 \pm 2.4$ & 0.33 & $0.44^{\mathrm{a}}$ & $0.35^{\mathrm{a}}$ \\
\hline HDL-C & $-14.7 \pm 2.7$ & $-11.5 \pm 1.9$ & $-14.7 \pm 1.4$ & 0.07 & 0.07 & $0.14^{\mathrm{a}}$ \\
\hline
\end{tabular}

$P=P$-value for difference among pre-, peri- and postmenopausal groups using age and BMI as dependent variables (ANOVA); $B 1, r$-value for age; $B 2, r$-value for weight.

${ }^{\text {a }} P<0.05$. 


\subsection{Body weight in premenopausal and postmenopausal women}

Weight gain over time has been observed pre, peri and post-menopausally [34,35], and menopause accelerates the inverse distribution in central obesity [36]. Our study showed that, during the follow-up period, women who were already postmenopausal had the least gain in BMI, similar to the finding of Pasquali et al. [34]. But, Matthews et al. [12] did not find BMI and weight changes during the transition to menopause, in contrast to the changes in women who remained premenopausal. However, they did not investigate the BMI change of women who were the same age and in menopause. The body weights and BMIs of our postmenopausal women changed very little. The results of Lindquist et al. [13] were closer to ours. We found the changes of TG and LDL-C levels were both age- and BMI-dependent; and the change of TC level was agedependent.

\section{Conclusions}

This study followed up changes occurring in three female subcohorts based on their menopausal status. Complete lipid profile data from pre, peri, and postmenopausal women were not yet available for every subcohort. Some cohort effects may still exist in the data. However, it can still be concluded that the high lipid profile in postmenopausal women is due to aging and weight gain.

Of the changes in lipid profile that occur during the transition to menopause, the increase in TC levels is the only significant change resulting from menopause. Elevation of TC, though influenced by age, is an independent anthropometric variable, which changes with menopausal status. Levels of LDL-C were age, BMI and menstruation-status-dependent. LDL-C does not increase in postmenstrual women. Age and obesity cause LDL-C levels to increase further before and decline after menopause. Decline in HDL-C is of concern.

\section{Acknowledgements}

This work was supported by contract numbers DOH79-06, DOH-80-30, DOH-83 and DOH-84 the Department of Health, Republic of China.

\section{References}

[1] Colditz GA, Willett WC, Stampfer MJ, Rosner B, Speizer FE, Hennekens $\mathrm{CH}$. Menopause and the risk of coronary heart disease in women. New Engl J Med 1987;316:1105-10.
[2] Kannel WB, Wilson PWF. Risk factors that attenuate the female coronary disease advantage. Arch Intern Med 1995;155:57-61.

[3] Campos H, McNamara JR, Wilson PWF, Ordovas JM, Schaefer EJ. Differences in low density lipoprotein subfractions and apolipoproteins in premenopausal and postmenopausal women. J Clin Endocrinol Metab 1988;67:30-5.

[4] Brown SA, Hutchinson R, Morrisett J, Boerwinkle E, Davis CE, Gotto AM Jr, Patsch W. Plasma lipid, lipoprotein cholesterol and apoprotein distributions in selected US communities. The atherosclerosis risk in communities (ARIC) study. Arterioscler Thromb 1993;13:1139-58.

[5] Stevenson JC, Crook D, Godsland IF. Influence of age and menopause and serum lipid and lipoproteins in healthy women. Atherosclerosis 1993;98:83-90.

[6] Lyu LC, Shieh MJ, Ordovas JM, Lichtenstein AH, Wilson PW, Schaefer EJ. Plasma lipoprotein and apolipoprotein level in Taipei and Framingham. Arterioscler Thromb 1993;13:1429-40.

[7] Pasquali R, Casimirri F, Pascal G, Tortelli O, Morselli Labate AM, Bertazzo D, Vicennati V, Gaddi A. Influence of menopause on blood cholesterol levels in women: the role of body composition, fat distribution and hormonal milieu. J Intern Med 1997;241:195-203.

[8] Ushiroyama T, Okamoto Y, Sugimoto O. Plasma lipid and lipoprotein levels in perimenopausal women. Acta Obstet Gynecol Scand 1993;72:428-33.

[9] Ko GT, Chan JC, Lau EM, Yueng VT, Chow CC, Wai HP, Li JK, So WY, Cockram CS. The effect of age on cardiovascular risk factors in Chinese women. Int J Cardiol 1997;61:221-7.

[10] Peters HW, Westendorp IC, Hak AE, Grobbee DE, Stehouwer CD, Hofman A, Witteman JC. Menopausal status and risk factors for cardiovascular disease. J Intern Med 1999;246:521-8.

[11] Torng PL, Su TC, Sung FC, Chien KL, Huang SC, Chow SN, Lee YT. Effect of menopause and obesity on lipids in middleaged Taiwanese women. Atherosclerosis 2000;153:413-21.

[12] Matthews KA, Meilahn E, Kuller LH, Kelsey SF, Caggiula AW, Wing RR. Menopause and risk factors for coronary heart disease. New Engl J Med 1989;321:641-6.

[13] Lindquist O. Intraindividual changes of blood pressure, serum lipids, and body weight in relation to menstrual status: results from a prospective population study of women in Goteborg, Sweden. Prev Med 1982;11:162-72.

[14] Akahoshi M, Soda M, Nakashima E, Shimaoka K, Seto S, Yano $\mathrm{K}$. Effects of menopause on trends of serum cholesterol, blood pressure, and body mass index. Circulation 1996;94:61-6.

[15] Poehlman ET, Toth MJ, Ades PA, Rosen CJ. Menopause-associated changes in plasma lipids, insulin-like growth factor 1 and blood pressure: a longitudinal study. Eur J Clin Invest 1997;27:322-6.

[16] Health and Vital Statistics, 1995. Department of Health. Executive Yuan, Republic of China, Taipei, Taiwan. 1995:134-47.

[17] Chien KL, Lee YT, Sung FC, Hsu HC, Su TC, Lin RS. Hyperinsulinemia and related atherosclerotic risk factors in the population at cardiovascular risk: a community-based study. Clin Chem 1999;45:938-46.

[18] Lee YT, Lin RS, Sung FC, Yang CY, Chien KL, Chen WJ, Su TC, Hsu HC, Huang YC. Chin-Shan community cardiovascular cohort in Taiwan-baseline data and five-year follow-up morbidity and mortality. J Clin Epidemiol 2000;53:838-46.

[19] Van Beresteijn EC, Korevaar JC, Huijbregts PC, Schouten EG, Burema J, Kok FJ. Perimenopausal increase in serum cholesterol: a 10-year longitudinal study. Am J Epidemiol 1993;127:383-92.

[20] Do KA, Green A, Guthrie JR, Dudley EC, Burger HG, Dennerstein L. Longitudinal study of risk factors for coronary heart disease across the menopausal transition. Am $\mathbf{J}$ Epidemiol 2000;151:584-93. 
[21] Longcope C, Herbert PN, McKinlay SM, Goldfield SR. The relationship of total and free estrogens and sex hormone-binding globulin with lipoproteins in women. J Clin Endocrinol Metab 1990;71:67-72.

[22] Shelley JM, Green A, Smith AM, Dudley E, Dennerstein L, Hopper J, Burger H. Relationship of endogenous sex hormones to lipids and blood pressure in mid-aged women. Ann Epidemiol 1998;8:39-45.

[23] Cauley JA, Gutai JP, Kuller LH, Powell JG. The relation of endogenous sex steroid hormone concentrations to serum lipid and liporpotein levels in postmenopausal women. Am J Epidemiol 1990;132:884-94.

[24] Wang TD, Lee CM, Wu CC, Lee TM, Chen WJ, Chen MF, Liau CS, Sung FC, Lee YT. The effects of dyslipidemia on left ventricular systolic function in patients with stable angina pectoris. Atherosclerosis 1999;146:117-24.

[25] Aldercreutz H, Mazur W. Phyto-oestrogens and Western diseases. Ann Med 1997;29:95-120.

[26] Wang JS, Carson EC, Lapane KL, Eaton CB, Gans KM, Lasater TM. The effect of physician office visits on CHD risk factor modification as part of a worksite cholesterol screening program. Prev Med 1999;28:221-8.

[27] Chow SN, Huang CC, Lee YT. Demographic characteristics and medical aspects of menopausal women in Taiwan. J Formos Med Assoc 1997;96:801-11.

[28] Van Ittersum FJ, van Baal WM, Kenemans P, Mijatovic V, Donker AJ, van der Mooren MJ, Stehouwer CD. Ambulatorynot office-blood pressure decline during hormone replacement therapy in healthy postmenopausal women. Am J Hypertens 1998;11:1147-52.
[29] Mercuto G, Zoncu S, Pano D, Pilia I, Lao A, Melis GB, Cherchi A. Estradiol-17 beta reduces blood pressure and restores the normal amplitude of the circadian blood pressure rhythm in postmenopausal hypertension. Am J Hypertens 1998;11:909-13.

[30] De Meersman RE, Zion AS, Giardina EG, Weir JP, Lieberman JS, Downey JA. Estrogen replacement, vascular distensibility, and blood pressure in postmenopausal women. Am J Physiol 1998;274:H1539-44.

[31] Lee M, Giardina EG, Homma S, DiTullio MR, Sciacca RR. Lack of effect of estrogen on rest and treadmill exercise in postmenopausal women without known cardiac disease. Am J Cardiol 1997;80:793-7.

[32] Hunt BE, Davy KP, Jones PP, DeSouza CA, Van Pelt RE, Tanaka H, Seals DR. Systemic hemodynamic determinants of blood pressure in women: age, physical activity, and hormone replacement. Am J Physiol 1997;273:H777-85.

[33] Burger HG. The endocrinology of menopause. J Steroid Biochem Mol Biol 1999;69:31-5.

[34] The VMH Collaborative Group, Pasquali R, Casimirri F, Labate AM, Tortelli O, Pascal G, Anconetani B, Flamia R, Capelli M, Barbbara L. Body weight, fat distribution and menopausal status in women. Int J Obes Relat Metab Disord 1994;18:61421.

[35] Wing RR, Matthews KA, Kuller LH, Meilahn EN, Plantinga PL. Weight gain at the time of menopause. Arch Intern Med 1991;151:97-102.

[36] Tohernof A, Poehlman ET. Effects of menopause transition on body fatness and body fat distribution. Obes Res 1998;6:246-54. 